\title{
Nondensity of stability for polynomial automorphisms of $\mathbb{C}^{2}$
}

\section{Gregery T. Buzzard}

\begin{abstract}
In the space of polynomial automorphisms of $\mathbb{C}^{2}$, the set of structurally stable maps is not dense. To obtain this result we develop some of the theory of moduli of stability for holomorphic maps. Also, the set of hyperbolic maps is not dense in the set of polynomial automorphisms; however, given any polynomial automorphism, $F$, of $\mathbb{C}^{2}$, there is a polynomial automorphism, $G$, of the same degree as and arbitrarily near $F$ such that each periodic point of $G$ is hyperbolic.
\end{abstract}

1. Introduction In this paper we consider questions of dynamical stability; i.e., when do small changes in a map lead to small changes in the dynamics? More precisely, let $\mathcal{G}$ be a topological space of diffeomorphisms of a manifold $M$. A map $F \in \mathcal{G}$ is structurally stable if there exists a neighborhood $\mathcal{U}$ of $F$ such that for each $G \in \mathcal{U}, F$ and $G$ are conjugate. In the study of dynamics of one complex variable, the question of the density of stability was resolved in [MSS] and [MS], where it was shown that in a family of rational maps of the Riemann sphere which depends holomorphically on a parameter, the set of structurally stable maps is dense. In contrast, for general $C^{k}$ diffeomorphisms of a compact surface, the set of structurally stable maps is not dense in most families; e.g., [I, Ch. 7, Sec. IV].

In this paper, we show that the situation for polynomial automorphisms of $\mathbb{C}^{2}$ of fixed degree is analogous to that for $C^{k}$ diffeomorphisms of a compact surface: structural stability is not dense. In doing so, we develop some of the theory of moduli of stability along the lines of [NPT]. In this particular case, there are numerical invariants associated with homoclinic and heteroclinic tangencies which can prevent conjugation between maps having different values for 
these invariants. We also show that hyperbolic maps are not dense in the space of polynomial automorphisms of sufficiently high degree, but that given a polynomial automorphism of degree $d$ having nontrivial dynamics, there is a nearby automorphism of the same degree such that all of its periodic points are hyperbolic. This latter result is one half of the Kupka-Smale Theorem in the setting of polynomial automorphisms.

Friedland and Milnor initiated the study of the dynamics of polynomial automorphisms of $\mathbb{C}^{2}$ in $[\mathrm{FM}]$. There they distinguish between elementary automorphisms, which are polynomially conjugate to an automorphism of the form $(x, y) \mapsto(a x+p(y), c y+d)(p$ polynomial, $a, c \neq 0)$ and which have simple dynamics, and the remaining automorphisms, which are termed nonelementary and which do not have simple dynamics.

Bedford and Smillie [BS2] introduced the notion of the dynamical degree of a polynomial automorphism of $\mathbb{C}^{2}$. Letting $\operatorname{deg} F$ denote the maximum of the degrees of the polynomial map $F$, the dynamical degree is defined as

$$
d=d(F)=\lim _{n \rightarrow \infty}\left(\operatorname{deg} F^{n}\right)^{1 / n}
$$

where $F^{n}$ denotes the $n$th iterate of $F$. This degree is a conjugacy invariant, and any nonelementary polynomial automorphism of $\mathbb{C}^{2}$ is conjugate to an automorphism whose dynamical degree is equal to its degree as a polynomial. Moreover, the elementary polynomial automorphisms are exactly those which have dynamical degree 1.

Definition 1.1 Let $\mathcal{P}_{d}$ denote the set of polynomial automorphisms of $d y$ namical degree $d$.

The topology on $\mathcal{P}_{d}$ is that induced by the compact-open topology applied both to an automorphism and its inverse. With this topology, $\mathcal{P}_{d}$ is complete. This topology can also be induced by a complete metric.

The main theorem of this paper is the following.

Theorem 1.1 There exists $N \in \mathbb{Z}^{+}$sufficiently large such that if $d \geq N$, then the set of structurally stable maps in $\mathcal{P}_{d}$ is not dense in $\mathcal{P}_{d}$.

In proving the previous theorem, we develop some techniques which lead to the following result.

Theorem 1.2 Let $F \in \mathcal{P}_{d}, d \geq 1$, and let $\mathcal{U}$ be a neighborhood of $F$. Then there exists $G \in \mathcal{U}$ such that each periodic point of $G$ is hyperbolic.

As noted earlier, this result is one half of the Kupka-Smale Theorem for polynomial automorphisms. It is an open question if the part of the Kupka-Smale Theorem giving the transversality of stable and unstable manifolds is also true in 
this setting. The full Kupka-Smale Theorem holds in the space of all holomorphic automorphisms of $\mathbb{C}^{n}[\mathrm{~B} 2]$, but the techniques used there to prove transversality involve composing a given automorphism with other automorphisms constructed to move the stable manifold in a specified manner. Doing this within $\mathcal{P}_{d}$ requires that all the additional automorphisms are linear, which is not sufficient for the argument in [B2].

In the next section we show that the order of contact between two 1-dimensional complex manifolds in $\mathbb{C}^{2}$ is a topological invariant, then construct a family of automorphisms whose fixed points have associated eigenvalues which are nonconstant functions of the parameter. In the final section we adapt the methods of [NPT] to the complex case to show that some differential information is preserved even under topological conjugacy, thus giving rise to moduli of stability. Combining these results with results of [B1] gives Theorem 1.1.

I am grateful to the referee for a careful reading of this paper and for several helpful suggestions. This research was supported in part by a grant from the National Science Foundation.

\section{Preliminary results}

Definition 2.1 Let $F$ be a smooth map of a manifold $M$. A point $p \in M$ is said to be nonwandering for $F$ if for each neighborhood $V$ of $p$, there exists $n>0$ such that $F^{n}(V) \cap V \neq \emptyset$. The nonwandering set of $F, \Omega=\Omega(F)$ is the set of all nonwandering points of $F$.

Recall that a periodic point $p$ of period $n$ of a diffeomorphism $F$ is hyperbolic if none of the eigenvalues of $D_{p} F^{n}$ has modulus 1 . Moreover, a hyperbolic periodic point has stable and unstable manifolds, and these manifolds are immersed 1dimensional complex manifolds when $F$ is holomorphic. Recall also that $F \in \mathcal{P}_{d}$ is hyperbolic if there is a continuous, $F$-invariant splitting $E^{s} \oplus E^{u}=T \mathbb{C}^{n} \mid \Omega$ and constants $C>0,0<\mu<1$ such that $\left\|D F^{n} \mid E^{s}\right\| \leq C \mu^{n}$ and $\left\|D F^{-n} \mid E^{u}\right\| \leq$ $C \mu^{n} \mid$ for $n \geq 0$. See [R] for further background in the real case.

The following theorem on the nondensity of hyperbolicity is an analytic counterpart of Theorem 1.1, which is topological in nature. It should be noted that the density of hyperbolicity for polynomials of one variable is an open question for every degree $d \geq 2$.

Theorem 2.1 There exists $N \in \mathbb{Z}^{+}$such that if $d \geq N$, then the set of hyperbolic maps is not dense in $\mathcal{P}_{d}$.

Proof. From [B1], there exists $N \in \mathbb{Z}^{+}$such that if $d \geq N$, then there is an open set $\mathcal{U} \subseteq \mathcal{P}_{d}$ such that each $F \in \mathcal{U}$ has a tangency between the stable and unstable manifolds for a basic set, $\Lambda$. Such a point of tangency, $p$, is in $\Omega(F)$, but it is immediate that such a point prevents a hyperbolic splitting of $\Omega(F)$. Since this is true for all maps in $U$, we see that hyperbolicity is not dense in $\mathcal{P}_{d}$ for $d$ large. 
The next lemma implies that the order of tangency between two 1-dimensional complex manifolds in $\mathbb{C}^{2}$ is a topological invariant.

Lemma 2.1 Suppose $M_{1}$ and $M_{2}$ are two 1-dimensional complex submanifolds of $\mathbb{C}^{2}$ which intersect in exactly one point $p$. Let $U$ be a neighborhood of $p$, and suppose that $\varphi: U \rightarrow \mathbb{C}^{2}$ is continuous and injective and that $\varphi\left(M_{j}\right)$ is also a complex manifold, $j=1,2$. Then the order of contact between $\varphi\left(M_{1}\right)$ and $\varphi\left(M_{2}\right)$ is the same as that between $M_{1}$ and $M_{2}$.

Proof. Using a topological change of coordinates, we may assume that $p$ and $\varphi(p)$ are both the origin, that $M_{1}$ and $\varphi\left(M_{1}\right)$ are contained in $\mathbb{C} \times\{0\}$, and that $M_{2}$ and $\varphi\left(M_{2}\right)$ are contained in the graphs of $z \mapsto z^{m}$ and $z \mapsto z^{n}$, respectively, for some positive integers $m$ and $n$.

Let $\Delta$ be the unit disk in $\mathbb{C}$ and $\Delta^{*}=\Delta \backslash\{0\}$. Consider an element in the fundamental group $\pi_{1}\left(\Delta \times \Delta^{*}\right)$ of the form $\gamma(\theta)=\left(\delta e^{i \theta}, \delta e^{i m \theta}\right)$, for small $\delta>0$. Then $[\gamma]=[m] \in \pi_{1}\left(\Delta \times \Delta^{*}\right)$, and since $\varphi$ is a homeomorphism of a neighborhood of the origin which preserves the $z$-axis, $[\varphi \circ \gamma]=[m] \in \pi_{1}\left(\Delta \times \Delta^{*}\right)$. From the topological change of coordinates introduced above, the image of $\varphi \circ \gamma$ is contained in the graph of $z \mapsto z^{n}$ minus the origin. In particular, the projection of $\varphi \circ \gamma$ to the $z$-axis is an element of $\pi_{1}\left(\Delta^{*}\right)$, and taking the graph of this projection under $z \mapsto z^{n}$ shows that $\varphi \circ \gamma \in n \mathbb{Z}$ in $\pi_{1}\left(\Delta \times \Delta^{*}\right)$. Thus $m \in n \mathbb{Z}$, and by a symmetric argument we have $n \in m \mathbb{Z}$, so $n=m$ as desired.

In the following proposition, $J F$ represents the determinant of the Jacobian matrix of partial derivatives of $F$. Note that $J F$ is constant for a polynomial automorphism $F$.

Proposition 2.1 Let $F \in \mathcal{P}_{d}$ with $d \geq 2$. Then there exists a one parameter family $\left\{F_{\mu}\right\}_{\mu \in \mathbb{C}^{*}} \subseteq \mathcal{P}_{d}$ with $F_{1}=F$ such that for all $n \geq 1$ there exists a discrete subset $E_{n} \subseteq \mathbb{C}^{*}$ such that if $\mu \in \mathbb{C}^{*} \backslash E_{n}$, then $F_{\mu}^{n}$ has exactly $d^{n}$ distinct fixed points, each of which varies holomorphically with $\mu$. For each such fixed point $p(\mu)$, the eigenvalues of $D_{p(\mu)} F^{n}$ are distinct and are nonconstant holomorphic functions of $\mu$. Also, $J F_{\mu}$ is independent of $\mu$.

Proof. Using $[\mathrm{FM}]$, we may assume that $F$ is a composition of generalized Hénon maps of the form $H_{j}(z, w)=\left(w, p_{j}(w)-a_{j} z\right)$, where $p_{j}$ is a monic polynomial of degree $d_{j} \geq 2$ and $a_{j} \neq 0$.

Suppose that

$$
D_{(0,0)} F=\left(\begin{array}{cc}
\alpha & \beta \\
\gamma & \delta
\end{array}\right) .
$$

For $p \in \mathbb{C}^{2}$, let $\psi_{p}(z, w)=(z, w)+p$. Replacing $F$ by $\psi_{p}^{-1} F \psi_{p}$ for some $p$ near 0 , we may assume that $\delta \neq 0$. We construct $F_{\mu}$ with this new $F$, then the final family is given by $\psi_{p} F \psi_{p}^{-1}$. 
For $\mu \in \mathbb{C}^{*}$, let $\varphi_{\mu}(z, w)=(\mu z, w / \mu)$, and let $F_{\mu}=\varphi_{\mu} F \varphi_{\mu}$. Since $\varphi_{\mu}$ is volume preserving, the Jacobian of $F_{\mu}$ is independent of $\mu$.

From [BS1], there exists $R>0$ such that for each $j$ and each $r \geq R$, the set $V_{r}^{+}=\{(z, w):|w| \geq r,|z| \leq|w|\}$ satisfies $H_{j}\left(V_{r}^{+}\right) \subseteq V_{2 r}^{+}$, and likewise $V_{r}^{-}=\{(z, w):|z| \geq r,|w| \leq|z|\}$ satisfies $H_{j}^{-1}\left(V_{r}^{-}\right) \subseteq V_{2 r}^{-}$. In particular, this implies that the nonwandering set of $F$ (hence the set of periodic points) is contained in $\Delta^{2}(0 ; R)$. A simple check shows that for $|\mu|<1, F_{\mu}\left(V_{|\mu| R}^{+}\right) \subseteq V_{2|\mu| R}^{+}$ and $F_{\mu}^{-1}\left(V_{|\mu| R}^{-}\right) \subseteq V_{2|\mu| R}^{-}$, and hence all periodic points for $F_{\mu}$ are contained in $\Delta^{2}(0 ;|\mu| R)$.

Let $\lambda_{1}, \lambda_{2}$ be the eigenvalues of $D_{(0,0)} F_{\mu}$ with $\left|\lambda_{1}\right| \leq\left|\lambda_{2}\right|$. Calculating $\lambda_{1}$ and $\lambda_{2}$ and using $\alpha \delta-\beta \gamma \neq 0$, we see that $\lim _{\mu \rightarrow 0} \lambda_{1}=0$ and $\lim _{\mu \rightarrow 0} \lambda_{2}=\infty$, since $\delta \neq 0$.

Hence for $\mu$ sufficiently near 0 , the eigenvalues of $D_{(0,0)} F_{\mu}$ are distinct and have modulus different from 1. Moreover, since each fixed point, $p(\mu)$, of $F_{\mu}^{n}$ is contained in $\Delta^{2}(0 ;|\mu| R)$, the derivative $D_{p(\mu)} F_{\mu}^{n}$ will be nearly $\left(D_{(0,0)} F_{\mu}\right)^{n}$ for $\mu$ near 0 . Since $F_{\mu}^{n}$ has at most $d^{n}$ fixed points by $[\mathrm{FM}]$, we can choose $\mu$ sufficiently near 0 that all of the fixed points of $F_{\mu}^{n}$ are hyperbolic with distinct eigenvalues.

From $[\mathrm{FM}], F_{\mu}^{n}$ has exactly $d^{n}$ fixed points counted with multiplicity, and since a hyperbolic fixed point has multiplicity 1 by the inverse function theorem, the argument just given implies that $F_{\mu}^{n}$ has $d^{n}$ distinct fixed points for $\mu$ sufficiently near 0 . In fact, for all $\mu$ outside a discrete subset of $\mathbb{C}, F_{\mu}^{n}$ has $d^{n}$ distinct fixed points. To see this, note that the set $A=\left\{(z, w, \mu): F_{\mu}^{n}(z, w)=(z, w)\right\}$ is a 1 -dimensional analytic set in $\mathbb{C}^{2} \times \mathbb{C}^{*}$ and that the projection of $A$ to $\mathbb{C}^{*}$ is proper with finite fibers. Since the cardinality of the fibers is constant except possibly on a 0 -dimensional analytic subset where one of the eigenvalues of a fixed point of $F_{\mu}^{n}$ is 1 , we see that for $\mu$ outside a discrete set, $F_{\mu}^{n}$ has $d^{n}$ distinct fixed points. For such $\mu$, the implicit function theorem implies that each fixed point varies holomorphically with $\mu$.

The sum of the eigenvalues of $D F_{\mu}^{n}$ is a holomorphic function on $\mathbb{C}^{2} \times \mathbb{C}^{*}$, hence on $A$. Since the product of the eigenvalues is $J F_{\mu}^{n}$, which is constant, it follows that each eigenvalue is a holomorphic function of $\mu$ whenever the two eigenvalues are distinct and $A$ is unbranched. Removing a zero-dimensional set, $E_{n}$, of $\mu$ 's at which any fixed point has an associated eigenvalue equal to 1 or any eigenvalue is repeated, we are left with an open dense set of $\mu$ 's which is the complement of a discrete set and on which each eigenvalue of each fixed point of $F_{\mu}^{n}$ is a holomorphic function of $\mu$. In view of the calculation of $\lim _{\mu \rightarrow 0} \lambda_{j}$ given above, each eigenvalue is nonconstant on this set.

With the previous result, we can prove Theorem 1.2 as a corollary. 
Proof of Theorem 1.2. Composing with a linear contraction near the identity, we may assume that $|J F| \neq 1$. Moreover, if $F$ has dynamical degree 1 , then it is elementary in the sense of $[\mathrm{FM}]$, and a simple calculation shows that the the proper choice of the linear contraction makes all periodic points hyperbolic. Hence we may assume that $d \geq 2$.

With this modified $F$, let $F_{\mu}$ be as in the previous proposition. From that result, for each $n \geq 1$, there exists an open dense set $U_{n}=C^{*} \backslash E_{n}$ such that for each $\mu \in U_{n}, F_{\mu}^{n}$ has $d^{n}$ distinct fixed points, and the corresponding eigenvalues vary holomorphically with $\mu$ and are nonconstant functions of $\mu$. For each $n$, let $U_{n}^{\prime}$ denote the subset of $U_{n}$ such that for each $\mu \in U_{n}^{\prime}$, there exists a fixed point $p(\mu)$ of $F_{\mu}^{n}$ such that some eigenvalue of $D_{p(\mu)} F_{\mu}^{n}$ has modulus 1 . Since $U_{n}^{\prime}$ is a real-analytic subset of $U_{n}$ of codimension 1 , we see that $U_{n} \backslash U_{n}^{\prime}$ is open and dense in $U_{n}$, hence in $\mathbb{C}^{*}$. Taking the intersection of $U_{n} \backslash U_{n}^{\prime}$ over all $n \geq 1$ gives a dense $\mathcal{G}_{\delta}$ subset $V$ of $\mathbb{C}^{*}$, so in particular there exists $\mu \in V$ near enough to 1 that $F_{\mu}$ in $\mathcal{U}$. Taking $G=F_{\mu}$ gives the theorem.

Remark. Note that Proposition 2.1 gives a way to construct a hyperbolic automorphism using the family $F_{\mu}$. For $\mu$ near 0 , the nonwandering set for $F_{\mu}$ will be contained in $\Delta^{2}(0 ;|\mu| R)$. On this bidisk, $D F_{\mu}$ will map a cone field in the vertical direction into itself with a rate of expansion bounded above one, and likewise $D F_{\mu}^{-1}$ will map a cone field in the horizontal direction into itself with a rate of expansion bounded above 1 . This implies that $F_{\mu}$ is hyperbolic on its nonwandering set.

3. Moduli of stability and proof of Main Theorem. The next lemma establishes the existence of moduli of stability for holomorphic maps: for a homoclinic tangency, the ratio of the logs of the absolute values of the eigenvalues of the associated fixed point is preserved under topological conjugacy. The methods used here are similar to those found in [NPT]. As in that work, we state the results here in terms of a heteroclinic tangency.

For this lemma, let $F_{1}, F_{2}$ be holomorphic automorphisms of $\mathbb{C}^{2}$, let $p_{j}$ and $q_{j}$ be fixed saddle points for $F_{j}$, and suppose that there is a tangency between $W^{u}\left(p_{j}, F_{j}\right)$ and $W^{s}\left(q_{j}, F_{j}\right)$ at the point $r_{j}$. Let $U_{j}$ be a neighborhood of the orbit of $r_{j}$ under $F_{j}$ with $p_{j}, q_{j} \in U_{j}$, and suppose that $\varphi: U_{1} \rightarrow U_{2}$ is a homeomorphism satisfying $\varphi\left(p_{1}\right)=p_{2}, \varphi\left(q_{1}\right)=q_{2}, \varphi\left(r_{1}\right)=r_{2}$, and $\varphi \circ F_{1}=F_{2} \circ \varphi$ on $U_{1} \cap F_{1}^{-1}\left(U_{1}\right)$, so that $\varphi$ gives a local conjugacy between $F_{1}$ and $F_{2}$. In particular, since the stable and unstable manifolds are topological objects, $\varphi$ maps $W^{s}\left(p_{1}, F_{1}\right) \cap U_{1}$ to $W^{s}\left(p_{2}, F_{2}\right) \cap U_{2}$, and likewise for the unstable manifolds.

Lemma 3.1 Let $\alpha_{j}$ be the contracting eigenvalue of $D_{p_{j}} F_{j}$ and let $\beta_{j}$ be the expanding eigenvalue of $D_{q_{j}} F_{j}$. Then

$$
\frac{\log \left|\alpha_{1}\right|}{\log \left|\beta_{1}\right|}=\frac{\log \left|\alpha_{2}\right|}{\log \left|\beta_{2}\right|} .
$$


Proof. Note that the stable and unstable manifolds have only a finite order of contact since otherwise they would agree on an open set, hence everywhere, which would imply that their union is a compact complex manifold contained in $\mathbb{C}^{2}$, which is impossible. In particular, Lemma 2.1 applies.

Consider a sequence $r_{1, k} \rightarrow r_{1}$ with $r_{1, k} \notin W^{u}\left(p_{1}\right) \cup W^{s}\left(q_{1}\right)$. Dropping to a subsequence, we may assume that $F_{1}^{-n_{k}}\left(r_{1, k}\right)$ converges to a point $p_{1}^{\prime} \in$ $W^{s}\left(p_{1}\right) \backslash\left\{p_{1}\right\}$ and that $F_{1}^{m_{k}}\left(r_{1, k}\right)$ converges to a point $q_{1}^{\prime} \in W^{u}\left(q_{1}\right) \backslash\left\{q_{1}\right\}$ with $p_{1}^{\prime}, q_{1}^{\prime} \in U_{1}$.

Let $V_{1}^{u}$ be a compact neighborhood of $r_{1}$ in $W^{u}\left(p_{1}\right)$ and $V_{1}^{s}$ a compact neighborhood of $r_{1}$ in $W^{s}\left(q_{1}\right)$. Let $d$ denote Euclidean distance, and let $d_{1, k}^{u}=$ $d\left(r_{1, k}, V_{1}^{u}\right)$ and $d_{1, k}^{s}=d\left(r_{1, k}, V_{1}^{s}\right)$, both of which converge to 0 as $k \rightarrow \infty$. Again dropping to a subsequence, we may assume that $d_{1, k}^{u} / d_{1, k}^{s}$ converges to $L \in[0, \infty]$ and that $m_{k} \geq n_{k}$.

Using Section 7.3 of [dMvS], we see that $F_{1}$ is $C^{1}$-linearizable in neighborhoods of $p_{1}$ and $q_{1}$. Hence for some constant $c>1$ independent of $k$, we have $\left|\alpha_{1}\right|^{n_{k}} / c \leq d_{1, k}^{u} \leq c\left|\alpha_{1}\right|^{n_{k}}$ and $\left.\left|\beta_{1}\right|^{-m_{k}}\left|/ c \leq d_{1, k}^{s} \leq c\right| \beta_{1}\right|^{-m_{k}}$. Taking log and keeping track of signs, we see that for $k$ large,

$$
\frac{n_{k} \log \left|\alpha_{1}\right|-\log c}{-m_{k} \log \left|\beta_{1}\right|+\log c} \geq \frac{\log d_{1, k}^{u}}{\log d_{1, k}^{s}} \geq \frac{n_{k} \log \left|\alpha_{1}\right|+\log c}{-m_{k} \log \left|\beta_{1}\right|-\log c} .
$$

By l'Hôpital's rule, the limit of the central term exists in $[0, \infty]$ and equals $1 / L$. On the other hand, the difference of the outer two terms tends to 0 as $k \rightarrow \infty$, so the limit of each of those terms also exists. Hence

$$
\lim _{k \rightarrow \infty} \frac{-m_{k}}{n_{k}}=L \frac{\log \left|\alpha_{1}\right|}{\log \left|\beta_{1}\right|} .
$$

Let $V_{2}^{s}=\varphi\left(V_{1}^{s}\right), V_{2}^{u}=\varphi\left(V_{1}^{u}\right), r_{2, k}=\varphi\left(r_{1, k}\right), d_{2, k}^{s}=d\left(r_{2, k}, V_{2}^{s}\right)$, and $d_{2, k}^{u}=$ $d\left(r_{2, k}, V_{2}^{u}\right)$. From Lemma 2.1, the order of tangency is the same for both pairs of manifolds, and we may put the tangencies in the normal form as in the proof of that lemma, so that the tangency is at the origin, the unstable manifold is the $z$-axis, and the stable manifold is the graph of $z \mapsto z^{n}$ for some $n \geq 2$.

To reach a contradiction, suppose first that $\log \left|\alpha_{1}\right| / \log \left|\beta_{1}\right|<\log \left|\alpha_{2}\right| / \log \left|\beta_{2}\right|$. We will show that then either

$$
\frac{d_{1, k}^{u}}{d_{1, k}^{s}} \rightarrow 0 \quad \text { or } \quad \frac{d_{2, k}^{u}}{d_{2, k}^{s}} \rightarrow \infty
$$

(or both). To see this, note that if $d_{2, k}^{u} / d_{2, k}^{s}$ is bounded from above, then there exists $C>0$ such that $\left|\alpha_{2}\right|^{n_{k}}\left|\beta_{2}\right|^{m_{k}} \leq C$. Hence

$$
\frac{\log \left|\alpha_{2}\right|}{\log \left|\beta_{2}\right|} \leq \frac{\log C}{n_{k} \log \left|\beta_{2}\right|}-\frac{m_{k}}{n_{k}}
$$


Choose $\delta>1$ such that $\left(\log \left|\alpha_{1}\right|+\log \delta\right) / \log \left|\beta_{1}\right|=\log \left|\alpha_{2}\right| / \log \left|\beta_{2}\right|$. Using this to replace the left hand side of $(3.2)$, we find

$$
n_{k} \log \left(\left|\alpha_{1}\right| \delta\right)+m_{k} \log \left|\beta_{1}\right| \leq \frac{\log C \log \left|\beta_{1}\right|}{\log \left|\beta_{2}\right|},
$$

so $\left(\left|\alpha_{1}\right| \delta\right)^{n_{k}}\left|\beta_{1}\right|^{m_{k}}$ is bounded and hence $\left|\alpha_{1}\right|^{n_{k}}\left|\beta_{1}\right|^{m_{k}} \rightarrow 0$. Given the bounds on $d_{1, k}^{u}$ in terms of $\left|\alpha_{1}\right|$ and on $d_{1, k}^{s}$ in terms of $\left|\beta_{1}\right|$, we see that $d_{1, k}^{u} / d_{1, k}^{s} \rightarrow 0$, as desired. Similarly, if $\log \left|\alpha_{1}\right| / \log \left|\beta_{1}\right|>\log \left|\alpha_{2}\right| / \log \left|\beta_{2}\right|$, then either $d_{1, k}^{u} / d_{1, k}^{s} \rightarrow$ $\infty$ or $d_{2, k}^{u} / d_{2, k}^{s} \rightarrow 0$.

Let $A^{s}=\left\{(z, w):\left|w-z^{n}\right| \leq|z|^{n} / 8\right\}, A^{u}=\left\{(z, w):|w| \leq|z|^{n} / 8\right\}$, and let $A^{0}$ be the closure of the complement of $A^{s} \cup A^{u}$. Note that for $r=(z, w)$ near $r_{1}, d\left(r, V_{1}^{s}\right) \approx\left|w-z^{n}\right|$, and $d\left(r, V_{1}^{u}\right) \approx|w|$, both up to a constant multiple which approaches 1 as $r$ tends to $r_{1}$. In particular, if $d_{1, k}^{u} / d_{1, k}^{s} \rightarrow 0$, then $r_{1, k} \in A^{u}$ for large $k$, while if $d_{1, k}^{u} / d_{1, k}^{s} \rightarrow \infty$, then $r_{1, k} \in A^{s}$ for large $k$. Hence, if $r_{1, k} \in A^{0} \cup A^{s}$ for all $k$, then $d_{1, k}^{u} / d_{1, k}^{s}$ is bounded below by a positive constant, while if $r_{1, k} \in A^{0} \cup A^{u}$ for all $k$, then $d_{1, k}^{u} / d_{1, k}^{s}$ is bounded above by a positive constant. Thus (3.1) implies that if $\log \left|\alpha_{1}\right| / \log \left|\beta_{1}\right|<\log \left|\alpha_{2}\right| / \log \left|\beta_{2}\right|$, then there exists a neighborhood $B_{1}$ of $r_{1}$ such that $\varphi\left(B_{1} \cap\left(A^{0} \cup A^{s}\right)\right) \subseteq A^{s}$. Likewise, if $\log \left|\alpha_{1}\right| / \log \left|\beta_{1}\right|>\log \left|\alpha_{2}\right| / \log \left|\beta_{2}\right|$, then there exists $B_{1}$ with $\varphi\left(B_{1} \cap\right.$ $\left.\left(A^{0} \cup A^{u}\right)\right) \subseteq A^{u}$.

To show the first case is impossible, let $\gamma_{1}(t)=\left(0, \epsilon e^{i t}\right)$. Then $\gamma_{1} \subseteq B_{1} \cap A^{0}$ for small $\epsilon$, and $\gamma_{1}$ is a generator for $\pi_{1}\left(\mathbb{C} \times \mathbb{C}^{*}\right)$. If $\varphi \circ \gamma_{1}(t) \subseteq A^{s}$, we can project along fibers parallel to the $w$-axis to $V_{2}^{s}$ to get a curve $\gamma_{2}$. Note that $\gamma_{2}$ misses the origin since the point of tangency is preserved, so $\gamma_{2}$ projects to the $z$-axis to give an element $[m] \in \pi_{1}\left(\mathbb{C}^{*}\right)$. Since $V_{2}^{s}$ is the graph of $z \mapsto z^{n}$ for some $n \geq 2$, we see that $\gamma_{2}=[m n] \in \pi_{1}\left(\mathbb{C} \times \mathbb{C}^{*}\right)$, so $\gamma_{2}$ is not a generator and hence $\varphi \circ \gamma_{1}$ is not a generator, contradiction. Thus $\varphi\left(B_{1} \cap\left(A^{0} \cup A^{s}\right)\right) \nsubseteq A^{s}$.

To show the second case is impossible, apply the map $(z, w) \mapsto\left(z, z^{n}-w\right)$ to both systems. This interchanges $A^{s}$ and $A^{u}$, so the preceding argument applies to show that $\varphi\left(B_{1} \cap\left(A^{0} \cup A^{u}\right)\right) \nsubseteq A^{u}$. Thus $\log \left|\alpha_{1}\right| / \log \left|\beta_{1}\right|=\log \left|\alpha_{2}\right| / \log \left|\beta_{2}\right|$ as desired.

Proof of Theorem 1.1. First we observe that by [B1], there exists $N \in \mathbb{Z}^{+}$ such that if $d \geq N$, then there is an open set $\mathcal{U} \subseteq \mathcal{P}_{d}$ and a dense subset $\mathcal{E} \subseteq \mathcal{U}$ such that each $F \in \mathcal{E}$ has a hyperbolic fixed point $p=p(F)$ with a homoclinic tangency: a tangency between the stable and unstable manifolds of $p$.

Fix $F \in \mathcal{U}$, and let $\mathcal{V}$ be any neighborhood of $F$ in $\mathcal{P}_{d}$. It suffices to find $F_{1}$ and $F_{2}$ in $\mathcal{V}$ which are not conjugate.

Choose $G \in \mathcal{V}$ with a homoclinic tangency associated to a fixed point $p$, and consider the family $G_{a}$ defined in Proposition 2.1. If there exists $G_{a} \in V$ 
with no homoclinic tangencies associated to a fixed point, then we are done by Lemma 2.1 since tangencies are preserved under conjugacy.

Otherwise, let $\alpha$ and $\beta$ be the contracting and expanding eigenvalues of $D_{p} G$, respectively. By Lemma 3.1, it suffices to show that there is a $\mu$ near 1 such that $G_{\mu}$ has no fixed point whose eigenvalues $\alpha(\mu)$ and $\beta(\mu)$ satisfy $\log |\alpha(\mu)| / \log |\beta(\mu)|=\log |\alpha| / \log |\beta|$. By Proposition 2.1, in any neighborhood of 1 there exists an open set, $U$, of parameters $\mu$ such that each $G_{\mu}$ has $d$ distinct fixed points, each of which has distinct associated eigenvalues which are nonconstant holomorphic functions of $\mu$. Let $p(\mu)$ be such a fixed point with contracting and expanding eigenvalues $\alpha(\mu)$ and $\beta(\mu)$, respectively. Suppose $\log |\alpha(\mu)| / \log |\beta(\mu)|$ equals a constant $C_{1}$ for some open set of $\mu$ 's. Since $\alpha(\mu) \beta(\mu)$ equals $J G_{\mu}$, which is a constant $C_{2}$ by Proposition 2.1, we can replace $\beta(\mu)$ by $C_{2} / \alpha(\mu)$ to obtain $\log |\alpha(\mu)|=C_{1} \log \left|C_{2}\right| /\left(1+C_{1}\right)$. This implies that $|\alpha(\mu)|$ and hence $\alpha(\mu)$ are constant, contradiction. Thus $\log |\alpha(\mu)| / \log |\beta(\mu)|$ is a nonconstant real-analytic function on $U$, so there is an open dense subset on which this function is not equal to $\log |\alpha| / \log |\beta|$. Taking the intersection of these open dense subsets corresponding to the $d$ distinct fixed points of $G_{\mu}$ shows that we can choose $\mu$ near 1 as claimed. Hence $F$ is not stable.

\section{REFERENCES}

[BS1] E. Bedford and J. Smillie, Polynomial diffeomorphisms of $\mathbb{C}^{2}$ : currents, equilibrium measure and hyperbolicity, Invent. Math., 103 (1991), no. 1, 69-99.

[BS2] E. Bedford and J. Smillie, Polynomial diffeomorphisms of $\mathbb{C}^{2}$ II: stable manifolds and recurrence, J. Am. Math. Soc., 4 (1991), 657-6799.

[B1] G. Buzzard, Infinitely many periodic attractors for holomorphic maps of 2 variables, Ann. of Math., 145 (1997), 389-417.

[B2] G. Buzzard, Kupka-Smale theorem for automorphisms of $\mathbb{C}^{n}$, Duke Math. J., 93 (1998), No. 3, 487-503.

[dMvS] W. De Melo and S. van Strien, Diffeomorphisms on surfaces with a finite number of moduli, Ergod. Th. Dyn. Sys., 7 (1987), no. 3, 415-462.

[FM] S. Friedland and J. Milnor, Dynamical properties of polynomial automorphisms, Ergod. Th. Dyn. Sys., 9 (1989), 67-99.

[I] M.C. IRwIN, Smooth Dynamical Systems, Academic Press, New York, 1980.

[MSS] R. Mañé, P. SAD, D. Sullivan, On the dynamics of rational maps, Ann. Sci. Ec. Norm. Sup., 16 (1983), 193-217.

[MS] C. McMullen, D. Sullivan, Quasiconformal homeomorphisms and dynamics, III, Adv. Math., 135 (1998), 351-395.

[NPT] S. Newhouse, J. Palis, F. Takens, Bifurcations and stability of families of diffeomorphisms, Inst. Hautes Etudes Sci. Publ. Math., 57 (1983), 5-71.

[R] C. Robinson, Dynamical systems : stability, symbolic dynamics, and chaos, CRC Press, Boca Raton, 1995. 